\title{
Scientometrics for Tech Mining
}

Selected papers presented at the 6th Annual Global TechMining Conference held in València (Spain) on 13 September 2016

Guest Editors:

Yi Zhang, Alan L. Porter and Denise Chiavetta 